\title{
Level Achieved in the Development of Skills for the Sexual and Reproductive Life of University Students After the Application of a Sexual Education Strategy
}

XVIII International Seminar on Health, Food and Human Nutrition

Corresponding Author:

Rosa Del Carmen Saeteros

Hernández

rsaeteros@espochedu.ec

Published: 9 September 2021

Production and Hosting by Knowledge E

(c) Rosa Del Carmen Saeteros Hernández et al. This article is distributed under the terms of the Creative Commons Attribution License, which permits unrestricted use and redistribution provided that the original author and source are credited.

\section{Nivel Conseguido en el Desarrollo de Habili- dades para la Vida Sexual y Reproductiva de Universitarios Posterior a la Aplicación de una Estrategia de Educación Sexual}

\author{
Rosa Del Carmen Saeteros Hernández ${ }^{1}$, Eida Ortiz Zayas ${ }^{2}$, and Angélica María \\ Saeteros Hernández ${ }^{3}$ \\ ${ }^{1}$ Carrera de Promoción de la Salud, Facultad Salud Pública, Escuela Superior Politécnica de \\ Chimborazo, Riobamba, Ecuador \\ ${ }^{2}$ Carrera de Medicina, Facultad Salud Pública, Escuela Superior Politécnica de Chimborazo, \\ Riobamba, Ecuador 3Municipio de Riobamba
}

\section{Abstract}

Introduction: Sexuality education must develop life skills. Methodology: The level of life skills was evaluated using a quasi- experimental design from before after with a control group random samples of 40 students were selected in the study and control group, respectively. The sex education strategy was implemented in the study group and a pre and post test was applied; before and after the strategy. Statistical techniques were used for dependent and independent samples, with a significance level of $p=0,05$. Results: The comparisons of the study and control groups before, the control group before and after, and the measurement after the implementation of the strategy of the satisfied needs of life skills in the study group, were within expectations, the most of the variables studied regarding the need to develop life skills are homogeneous in the study and control group before implementing the strategy; and no significant differences were found in the comparison of the control group before and after. Subsequent measurement regarding needs met or level achieved in the development of skills for sexual and reproductive life in the study group was high, even for some of them, $100 \%$ of students appreciated that they had achieved it. Conclusion: The sex education strategy was effective in developing skills for sexual and reproductive life due to the high level achieved by the students who participated in the strategy.

Keywords: education, sex, life skills, sexual and reproductive health.

\section{Resumen}

Introducción: La educación sexual debe desarrollar habilidades para la vida. Metodología: Se evaluó el nivel de habilidades para la vida mediante un diseño cuasi experimental de antes después con grupo control, se seleccionaron muestras aleatorias de 40 estudiantes en el grupo de estudio y control respectivamente, se implementó la estrategia de educación sexual en el grupo de estudio y se aplicó una pre y post prueba; antes y después de la estrategia. Se utilizaron técnicas estadísticas para muestras dependientes e independientes, 
con un nivel de significación $p=0,05$. Resultados: Las comparaciones de los grupos de estudio y control antes, grupo control antes y después y la medición posterior a la implementación de la estrategia de las necesidades satisfechas de habilidades para la vida en el grupo de estudio, estuvieron dentro de lo esperado, la mayoría de variables estudiadas respecto a la necesidad de desarrollar habilidades para la vida son homogéneas en el grupo de estudio y control antes de implementar la estrategia; y no se encontraron diferencias significativas en la comparación del grupo control antes y después. La medición posterior respecto a necesidades satisfechas o nivel conseguido en el desarrollo de habilidades para la vida sexual y reproductiva en el grupo de estudio fue elevado, incluso para algunas de ellas, el $100 \%$ de estudiantes apreció que lo había logrado. Conclusiones: La estrategia de educación sexual fue efectiva para desarrollar habilidades para la vida sexual y reproductiva debido al nivel elevado conseguido por los estudiantes que participaron en la estrategia.

Palabras Clave: educación sexual, habilidades para la vida, salud sexual, reproductiva.

\section{Introducción}

Los jóvenes representan el estrato de población más expuesto a los problemas de Salud Sexual y Reproductiva (SSR), así las Infecciones de Transmisión Sexual (ITS) incluido el $\mathrm{VIH}$ y Sida, los embarazos no planificados a temprana edad, los abortos en condiciones de riesgo, ocurren en el contexto del inicio temprano de las relaciones sexuales, donde son comunes las múltiples parejas sexuales, la infidelidad y otras condiciones como el consumo de alcohol y sustancias estupefacientes que agravan y aumentan el riesgo en su SSR.

Varios estudios en universitarios dan cuenta de los limitados e insuficientes conocimientos, aunque algunos estudios indican que tienen cierta información previa sobre salud sexual; se observa actitudes negativas, comportamientos inadecuados, fruto de la deficiencias en la educación sexual, valores impuestos, mitos, estereotipos, creencias falsas y la escasa o nula participación en eventos formativos [1-4].

La Constitución de la República del Ecuador 2008 [5], en el artículo 66, reconoce y garantiza el derecho a tomar decisiones libres, informadas, voluntarias y responsables sobre su sexualidad, su vida y orientación sexual, y declara que el estado promoverá el acceso a los medios necesarios para que estas decisiones se den en condiciones seguras. El artículo 347 numeral 4 de la carta fundamental indica: Que será responsabilidad del estado asegurar que todas las entidades educativas impartan una educación en ciudadanía, sexualidad y ambiente, desde el enfoque de derechos.

Existen evidencias científicas del impacto positivo de la educación sexual, según la orientación técnica dada por la Organización de Naciones Unidas para la Educación la Ciencia y la Cultura (UNESCO), en base a la revisión de 272 estudios que midieron el impacto de diversos programas en el comportamiento sexual, todos ellos han contribuido a enriquecer el conocimiento sobre diferentes aspectos de la sexualidad [6].

Los estudiantes universitarios transitan por una etapa crucial de consolidación de la identidad y manifestaciones de aumento de la responsabilidad, por tanto una fase de 
cambios en el proceso de desarrollo personal y necesidad de educación en cuanto al fomento de habilidades para la vida, definidas por la OMS [7], como 'aquellas destrezas que permiten que los adolescentes adquieran las aptitudes necesarias para su avance personal y para enfrentar en forma efectiva los retos de la vida diaria'; como: La autoconfianza para tomar decisiones autónomas, la comunicación asertiva para negociar las mejores prácticas, la mejora de la autoestima, la empatía, el respeto por la diferencia y la diversidad, la igualdad de derechos según el género, el manejo de conflictos, la capacidad para cuestionar hábitos poco saludables y el manejo de emociones y sentimientos. De esta manera podrán los jóvenes decidir en libertad y establecer y mantener relaciones interpersonales sanas enriquecedoras y positivas.

En la consulta global de la OMS, por medio de la Encuesta Mundial de Salud a Escolares (GSHS) diseñada en módulos alcohol y otras drogas, hábitos de alimentación, higiene, salud mental, actividad física, factores de protección, comportamientos sexuales que contribuyen al SIDA u otra ITS o embarazo no intencionado, los adolescentes exponen diferentes razones para adoptar estos comportamientos poco saludables: entre ellos disfrutar del comportamiento, haber formado un hábito que ahora es difícil de romper y la presión de los compañeros [8-10].

Frente a lo anteriormente expuesto, se han generado documentos, iniciativas y discusiones tanto en la conferencia mundial sobre educación para todos, foro mundial sobre la educación, acción Dakar, que concluyen en la necesidad de implementar educación sanitaria basada en la preparación para la vida activa, por tanto, la OMS desde 1993 ha promovido las habilidades para la vida definiéndolas como habilidades para el comportamiento adaptativo y positivo que permiten a las personas hacer frente a los problemas de la vida cotidiana de manera efectiva [10].

El modelo de habilidades para la vida ha sido implementado a nivel mundial fundamentalmente en proceso de prevención primaria de conductas de riesgos como consumo y abuso de sustancias psicoactivas en adolescentes y adultos jóvenes, se han encontrado algunas experiencias de su aplicación en la prevención de conductas sexuales de riesgo en adolescentes y adultos jóvenes [11].

El objetivo de esta investigación fue medir el nivel conseguido en el desarrollo de habilidades para la vida sexual y reproductiva, posterior a la aplicación de una estrategia de educación sexual.

\section{Metodología}

Para la evaluación del nivel conseguido de habilidades para la vida sexual y reproductiva posterior a la ejecución de estrategia de educación sexual, se utilizó un diseño cuasi experimental de antes después con grupo control.

Una investigación previa demostró que los grupos de estudio constituido por estudiantes de la Facultad de Salud Pública y del grupo control, pertenecientes a estudiantes del resto de facultades, de la Escuela Superior Politécnica de Chimborazo, fueron homogéneos en la mayoría de variables estudiadas, lo que los hace ser comparables, en este sentido de estos grupos originales, para la implementación y evaluación de la estrategia de educación sexual, se seleccionó grupos a pequeña escala, a partir de 
muestras aleatorias, 40 estudiantes de cada uno de los mentados grupos, cuando se seleccionaron los 40 estudiantes se compararon las principales características sociodemográficas para las que fueron homogéneos, la estrategia de educación sexual se implementó y evaluó en el grupo de estudio.

El instrumento utilizado para la evaluación de la estrategia de educación sexual fue en la modalidad de pre y post prueba, para la preprueba se incluyó la variable necesidad de desarrollar habilidades para la vida, la post prueba para el grupo de estudio tiene una variación en la pregunta respecto a habilidades para la vida, pues en ella se recoge información sobre las necesidades satisfechas en el desarrollo de habilidades para la vida. A fin de validar el pre y pos prueba, se realizó una prueba piloto de este instrumento con 30 estudiantes de la universidad que no participaron en esta fase de la investigación.

Tanto la necesidad de desarrollar habilidades para la vida sexual y reproductiva, como las necesidades satisfechas en el desarrollo de habilidades para la vida, fueron investigadas con 11 preguntas, con respuestas en categorías ordinales (mucho, poco y nada).

En el análisis estadístico se consideraron tres combinaciones. Para las comparaciones de los grupos de estudio y control antes de la implementación de la estrategia, se aplicaron técnicas estadísticas para muestras independientes. En el caso de las variables cualitativas se aplicó la prueba de homogeneidad, con el estadígrafo chi cuadrado de Pearson (X2) para las dicotómicas y chi cuadrado de Bartholomew para las ordinales.

Para la comparación antes y después en el grupo control, se emplearon técnicas para muestras relacionadas. En el caso de las variables cualitativas dicotómicas se aplicó la prueba de Mc Nemar, para las variables ordinales se usó la prueba de Stuart Maxwell.

En todas ellas se consideró un nivel de significación $p=0,05$.

Las necesidades satisfechas de habilidades para la vida en el grupo de estudio se midieron con porcentajes.

\section{Resultados}

La estrategia de educación sexual, se diseñó en base a las necesidades de educación sexual y reproductiva determinadas en una primera fase de la investigación, fue diseñada e implementada con metodología de pares, es decir por estudiantes de la propia universidad, de la carrera de Promoción de la Salud, quienes cursaban la cátedra de Salud Sexual, y el acompañamiento de expertos. Un elemento para destacar es la propuesta de los estudiantes de incorporar a la estrategia la promoción de las habilidades para la vida, aspecto que fue tomado en cuenta en el diseño y desarrollo de la misma.

En esta investigación se asumió como estrategia de educación sexual, al conjunto de acciones de enseñanza aprendizaje extracurricular, planificadas sistemáticamente, aplicadas en el entorno educativo politécnico, en base a las necesidades educativas 
determinadas mediante la investigación, para aportar al logro y mantenimiento de la SSR de estudiantes universitarios.

Contiene una serie de temas, que se desarrollan en sesiones y actividades educativas, con la finalidad de la reducción de información incorrecta, la construcción y deconstrucción de conocimientos, desmitificación de creencias y mitos, la consolidación de sentimientos, valores y actitudes positivas; generación de habilidades para la vida sexual y reproductiva y fomento de estilos de vida saludables, que promuevan cambios en las intenciones hacia comportamientos sexualmente saludables.

Pensar en ello significó movilizar una visión positiva de la SSR, y no solamente planear la transmisión de herramientas típicamente higienistas, de prevención o disminución riesgo; sino que integró el desarrollo y apropiación de habilidades sociales mucho más complejas, que les permita cumplir con los ideales en la sexualidad, en el contexto del amor y la afectividad, del disfrute y goce sexual, de la comunicación libre de prejuicios y temores, del respeto y reconocimiento a los Derechos Sexuales y Reproductivos, de la planificación familiar, de los valores, de la equidad de género y de la comprensión de las diferentes orientaciones sexuales.

La estrategia de educación sexual requirió del diseño de materiales de apoyo, como un libro del facilitador, un libro del estudiante, así como materiales promocionales como una camiseta un bolso y otros.

Cabe mencionar que la estrategia de educación sexual fue validada por expertos, y en relación al desarrollo de habilidades para la vida, consideraron que las mismas estuvieron en correspondencia con las posibilidades de educación sexual planteadas en la estrategia.

Las principales características de la estrategia de educación sexual fueron:

\subsection{Condiciones de la estrategia de educación sexual:}

1. Diseñada y aplicada con metodología de pares,

2. Basada en necesidades educativas identificadas en la investigación,

3. Apoyada en conocimientos científicos, realistas y sin prejuicios,

4. Basada en una pedagogía problematizadora,

5. Incluye una perspectiva de género que transversaliza cada tema, sesión y actividad,

6. Inspirada en los Derechos Sexuales y Reproductivos,

7. Llevada a cabo en actividades extracurriculares,

8. Fomenta habilidades para la vida sexual y reproductiva,

9. Es participativa y dinamizadora de la esfera afectiva, con el uso de técnicas de enseñanza aprendizaje interactivas,

10. Contempla la evaluación participativa del proceso, y de los resultados a corto plazo, es decir posterior a la implementación de la estrategia de educación sexual. 


\subsection{Objetivos de la estrategia de educación sexual}

1. Reducir información incorrecta, deconstruir conocimientos en salud sexual y reproductiva,

2. Desmitificar creencias, mitos y tabúes sobre la base del conocimiento científico en sexualidad,

3. Clarificar sentimientos, valores y actitudes positivas relacionadas con la esfera sexual,

4. Mejorar las intenciones hacia comportamientos saludables,

5. Mejorar la comunicación en temas de sexualidad,

6. Generar habilidades para la vida sexual y reproductiva,

7. Fomentar estilos de vida saludables.

\section{Resumen de los Módulos y objetivos de la estrategia de educación sexual}

\subsection{Sesión para iniciar}

\subsubsection{Conociéndonos y creando un ambiente democrático}

1. Posibilitar un ambiente de confianza e integración entre los participantes,

2. Socializar el proceso de capacitación, los contenidos y los aspectos logísticos de los eventos de capacitación,

3. Entregar los materiales de uso del participante (Bolso, camiseta del proyecto, cuaderno del estudiante),

4. Firmar las cartas de consentimiento informado de participar en la investigación,

5. Contar con los compromisos del grupo para el desarrollo de las diferentes actividades de capacitación.

\subsection{Módulo I}

\subsubsection{Sexualidad valores y derechos sexuales y reproductivos}

1. Identificar las características biopsicosociales, ético, legales relacionadas con la sexualidad,

2. Reconocer las dimensiones, funciones y componentes de la sexualidad,

3. Identificar los elementos de la Respuesta Sexual Humana en el varón y la mujer,

4. Identificar los derechos sexuales y reproductivos, 
5. Evidenciar una actitud positiva ante los valores de la sexualidad,

6. Respetar la vivencia de la sexualidad de las personas,

7. Valorar la individualidad sexual y las opciones que de ella se desprenden.

\subsection{Módulo II}

\subsubsection{Salud reproductiva}

1. Reconocer los mitos y las realidades del embarazo no planificado y sus riesgos,

2. Identificar los diferentes tipos de métodos anticonceptivos,

3. Asumir una actitud positiva frente a los métodos anticonceptivos,

4. Vencer los temores, resistencias y conflictos naturales acerca del uso de los métodos anticonceptivos,

5. Decidir con responsabilidad su paternidad y maternidad,

6. Analizar las ideas y sentimientos asociados a la posibilidad de tener un hijo durante los estudios en la politécnica,

7. Decidir con responsabilidad el momento oportuno de tener relaciones sexuales,

8. Analizar las ideas y los sentimientos asociados a la posibilidad de tener un hijo,

9. Utilizar habilidades para negociar el uso de anticonceptivos.

\subsection{Módulo III}

\subsubsection{Infecciones de transmisión sexual}

1. Reconocer los diferentes tipos de infecciones de transmisión sexual,

2. Identificar las formas de transmisión de las Infecciones de Transmisión sexual,

3. Reconocer a las estrategias de prevención y las conductas sexuales seguras o protegidas como indispensables para la prevención de las ITS,

4. Identificar los de signos síntomas comunes en las ITS.

\subsection{Módulo IV VIH - Sida}

1. Analizar las creencias negativas y positivas que existen sobre el tema y desmitificar los conceptos sobre el VIH-sida,

2. Manifestar conocimientos científicos acerca del VIH- Sida, las formas transmisión y no transmisión, 
3. Reflexionar sobre los pensamientos, creencias, opiniones y juicios de valor que se pueden generar alrededor del tema de $\mathrm{VIH}$-sida y el trato a las personas que viven con $\mathrm{VIH}$-sida,

4. Identificar las medidas de prevención contra el VIH-Sida,

5. Mostar cambios en actitudes y conductas para disminuir la propagación de la infección del VIH.

\subsection{Módulo V Condón}

1. Demostrar conocimiento en el uso y manejo adecuado del condón del varón y la mujer en las relaciones sexuales,

2. Identificar los mitos sobre el condón,

3. Demostrar habilidades en el uso correcto del condón del varón y la mujer,

4. Mostrar actitudes positivas para el uso del condón,

5. Evidenciar habilidades para la negociación del uso del condón en pareja.

\subsection{Módulo VI}

\subsubsection{Estilos de vida y sexualidad Asumiendo compromiso}

1. Evidenciar conocimiento y percepción del riesgo de los efectos reales del uso de drogas, alcohol y la sexualidad,

2. Identificar las conductas de riesgo en la vida sexual y reproductiva e iniciar un compromiso de cambio.

\section{Metodología de trabajo propuesta en el desarrollo de módulos/sesiones y actividades}

La metodología es activa-participativa, explicativa y analítica, en la cual se buscó despertar el interés sobre las temáticas y la satisfacción de aprendizaje de los estudiantes politécnicos.

Los estudiantes de la Escuela Superior Politécnica de Chimborazo son los principales autores en este proceso, porque sobre la base de las actividades planeadas en cada sesión se realizó una construcción colectiva de los conocimientos y su actitud permitirá analizar y reflexionar los temas que contribuirán a mejorar la vivencia de su sexualidad. Cada actividad tiene un espacio para el cierre y/o la evaluación de la sesión. Se aplicará mediante la participación de pares (estudiantes politécnicos) capacitados y la guía de la autora de la investigación. 


\section{Habilidades para la vida sexual y reproductiva a desar- rollar en las sesiones y actividades Autoconocimiento}

Incrementar los conocimientos sobre temas de sexualidad. Tener más recursos para con seguridad cumplir el ideal en la salud sexual y reproductiva. Cambiar para bien la forma de pensar sobre temas relacionados con sexualidad.

\section{Empatía}

Ser más tolerante en la interacción con los demás y comprender mejor las vivencias sexuales. Comunicación asertiva: Expresar con claridad lo que piensa, lo que siente o necesita en cuanto a la salud sexual y reproductiva.

\section{Relaciones interpersonales}

Ser capaz de manejar la presión de grupos de pares y mantener autonomía en las decisiones que se relacionan con la salud sexual y reproductiva.

\section{Toma de decisiones}

Escoger alternativas más seguras para mantener un nivel adecuado en la salud sexual y reproductiva.

\section{Manejo de problemas y conflictos}

Manejar adecuadamente los problemas y conflictos que pueden derivarse de la vivencia de la sexualidad.

\section{Pensamiento creativo}

Abordar la realidad de la salud sexual y reproductiva de forma novedosa y con originalidad.

\section{Pensamiento crítico}

Cambiar si es necesario sus comportamientos para conservar la salud sexual y reproductiva, que le permitan un crecimiento personal.

\section{Resultados de la evaluación de habilidades para la vida.}




\subsection{Necesidades de desarrollar habilidades para la vida sexual y reproductiva.}

\subsubsection{Grupo de estudio y control antes de la intervención con necesi- dades de desarrollar habilidades para la vida sexual y reproduc- tiva}

\section{Table 1}

Estudiantes de los grupos estudio y control antes de la intervención con necesidades de desarrollar habilidades para la vida sexual y reproductiva según variables exploradas.

\begin{tabular}{|c|c|c|c|c|c|}
\hline \multirow{3}{*}{$\begin{array}{l}\text { Necesidad de desarrollar habilidades para la } \\
\text { vida sexual y reproductiva }\end{array}$} & \multicolumn{4}{|c|}{ Grupos de investigación } & \multirow[t]{3}{*}{ Probabilidad } \\
\hline & \multicolumn{2}{|c|}{ Estudio } & \multicolumn{2}{|c|}{ Control } & \\
\hline & No. & $\% n=40$ & No. & $\% n=40$ & \\
\hline $\begin{array}{l}\text { Incrementar conocimientos sobre temas de } \\
\text { sexualidad }\end{array}$ & 37 & 92,5 & 34 & 85,0 & $>0,05^{b}$ \\
\hline $\begin{array}{l}\text { Cambiar para bien la forma de pensar sobre } \\
\text { temas relacionados con sexualidad }\end{array}$ & 33 & 82,5 & 30 & 75,0 & $>0,05^{b}$ \\
\hline $\begin{array}{l}\text { Tener más recursos para con seguridad } \\
\text { cumplir el ideal en la salud sexual y } \\
\text { reproductiva }\end{array}$ & 34 & 85,0 & 30 & 75,0 & $>0,05^{b}$ \\
\hline $\begin{array}{l}\text { Ser más tolerante en la interacción con los } \\
\text { demás y comprender mejor sus vivencias } \\
\text { sexuales }\end{array}$ & 28 & 70,0 & 24 & 60,0 & $>0,05^{b}$ \\
\hline $\begin{array}{l}\text { Expresar con claridad lo que piensa, lo que } \\
\text { siente o necesita en cuanto a la salud sexual } \\
\text { y reproductiva }\end{array}$ & 36 & 90,0 & 24 & 60,0 & $<0,005^{b}$ \\
\hline $\begin{array}{l}\text { Ser capaz de manejar la presión de grupos } \\
\text { de pares y mantener la autonomía en las } \\
\text { decisiones que se relacionan con salud } \\
\text { sexual yreproductiva }\end{array}$ & 33 & 82,5 & 20 & 50,0 & $<0,005^{b}$ \\
\hline $\begin{array}{l}\text { Escoger alternativas más seguras para } \\
\text { mantener un nivel adecuado en salud sexual } \\
\text { y reproductiva }\end{array}$ & 36 & 90,0 & 29 & 72,5 & $0,086^{a}$ \\
\hline $\begin{array}{l}\text { Manejar adecuadamente los problemas y } \\
\text { conflictos que pueden derivarse de la } \\
\text { vivenciade sexualidad }\end{array}$ & 36 & 90,0 & 32 & 80,0 & $>0,05^{b}$ \\
\hline $\begin{array}{l}\text { Cambiar los comportamientos sexuales que } \\
\text { le permitan un crecimiento personal }\end{array}$ & 35 & 87,5 & 31 & 77,5 & $0,377^{a}$ \\
\hline $\begin{array}{l}\text { Abordar la realidad de la salud sexual y } \\
\text { reproductiva de forma novedosa y con } \\
\text { originalidad }\end{array}$ & 38 & 95,0 & 32 & 80,0 & $<0,05^{b}$ \\
\hline $\begin{array}{l}\text { Cambiar comportamientos si es necesario, } \\
\text { para conservar la salud sexual y reproductiva. }\end{array}$ & 35 & 87,5 & 27 & 67,5 & $0,061^{a}$ \\
\hline
\end{tabular}

${ }^{a}$ Para X2 Pearson Se aplicó el estadígrafo X2 Pearson donde solo hubo respuestas en dos categorías (mucho y poco); ${ }^{b}$ para $\mathrm{X} 2$ Bartholomew.

Las necesidades para desarrollar habilidades para la vida sexual y reproductiva se muestran en la Tabla 1, donde se refleja la respuesta mucho en todas las variables. Los grupos estudio y control resultaron homogéneos en la mayoría de variables estudiadas, 
excepto la necesidad de expresar con claridad lo que piensa, lo que siente o necesita en cuanto a la SSR con un 90,0\% y 60,0\%, ser capaz de manejar la presión de grupos de pares y mantener la autonomía en las decisiones que se relacionan con salud sexual y reproductiva, $82,5 \%$ y $50,0 \%$ y abordar la realidad de la SSR de forma novedosa y con originalidad, un $95,0 \%$ y $80,0 \%$ respectivamente.

La menor frecuencia de respuesta mucho para el grupo estudio estuvo en la variable ser más tolerante en la interacción con los demás y comprender mejor sus vivencias sexuales con $70,0 \%$ y en el grupo control para ser capaz de manejar la presión de grupos de pares y mantener la autonomía en las decisiones que se relacionan con salud sexual y reproductiva con $50,0 \%$.

\subsubsection{Grupo control antes y después de la intervención con necesi- dades de desarrollar habilidades para la vida sexual y reproduc- tiva}

Se identificó el nivel de necesidad de desarrollar habilidades para la vida sexual y reproductiva, la Tabla 2 muestra las respuestas en la categoría mucho. En general los porcentajes de esta respuesta fueron semejantes entre los momentos, lo que hace que no se encontraran diferencias estadísticamente significativas. Solo se identificaron estas diferencias para expresar con claridad lo que piensa, lo que siente o necesita en cuanto a la salud sexual y reproductiva con $60,0 \%$ antes y $80,0 \%$ después; y ser capaz de manejar la presión de grupos de pares y mantener la autonomía en las decisiones que se relacionan con salud sexual y reproductiva con $50,0 \%$ y $70,0 \%$.

\subsubsection{Grupo de estudio, según nivel conseguido en el desarrollo de habilidades para la vida sexual y reproductiva}

En la Tabla 3, se observa el nivel conseguido en el desarrollo de habilidades para la vida sexual y reproductiva según la percepción de los estudiantes del grupo estudio luego de participar en la estrategia de educación sexual; todas las respuestas estuvieron por encima del 90,0\%; es de destacar que el 100,0\% asumió que ahora es capaz de expresar lo que siente o necesita en cuanto a su salud sexual y reproductiva, así como escoger alternativas sexuales más seguras para mantener un nivel adecuado en su salud sexual y reproductiva.

\section{Discusión}

El constructo habilidades para la vida fue propuesto por la OMS, en su proceso de difusión se ha fortalecido y ha sido operacionalizado agrupando las habilidades afines, es de resaltar que el enfoque desde sus inicios ha tenido una perspectiva educativa y el marco de referencia es amplia abordando componentes humanístico, cognitivo y conductual; la base teórica de habilidades para la vida, y sus reinterpretaciones ha permitido el diseño y la implementación de programas de intervención en población 
Table 2

Estudiantes del grupo control antes y después de la intervención con necesidades de desarrollar habilidades para la vida sexual y reproductiva según variables exploradas.

\begin{tabular}{|c|c|c|c|c|c|}
\hline \multirow{3}{*}{$\begin{array}{l}\text { Necesidad de desarrollar habilidades para la } \\
\text { vida sexual y reproductiva respuesta: Mucho }\end{array}$} & \multicolumn{4}{|c|}{ Grupo Control } & \multirow[t]{3}{*}{ Probabilidad } \\
\hline & \multicolumn{2}{|c|}{ Antes } & \multicolumn{2}{|c|}{ Después } & \\
\hline & No. & $\% n=40$ & No. & $\% n=40$ & \\
\hline $\begin{array}{l}\text { Incrementar conocimientos sobre temas de } \\
\text { sexualidad }\end{array}$ & 34 & 85,0 & 34 & 85,0 & $0,160^{b}$ \\
\hline $\begin{array}{l}\text { Cambiar para bien la forma de pensar sobre } \\
\text { temasrelacionados con sexualidad }\end{array}$ & 30 & 75,0 & 32 & 80,0 & $0,600^{b}$ \\
\hline $\begin{array}{l}\text { Tener más recursos para con seguridad } \\
\text { cumplir el idealen la salud sexual y } \\
\text { reproductiva }\end{array}$ & 30 & 75,0 & 32 & 80,0 & $0,195^{b}$ \\
\hline $\begin{array}{l}\text { Ser más tolerante en la interacción con los } \\
\text { demás ycomprender mejor sus vivencias } \\
\text { sexuales }\end{array}$ & 24 & 60,0 & 28 & 70,0 & $0,579^{b}$ \\
\hline $\begin{array}{l}\text { Expresar con claridad lo que piensa, lo que } \\
\text { siente onecesita en cuanto a la salud sexual y } \\
\text { reproductiva }\end{array}$ & 24 & 60,0 & 32 & 80,0 & $0,008^{a}$ \\
\hline $\begin{array}{l}\text { Ser capaz de manejar la presión de grupos } \\
\text { de pares ymantener la autonomía en las } \\
\text { decisiones que se relacionan con salud } \\
\text { sexual y reproductiva }\end{array}$ & 20 & 50,0 & 28 & 70,0 & $\begin{array}{l}0,012^{b} \\
0,007^{c}\end{array}$ \\
\hline $\begin{array}{l}\text { Escoger alternativas más seguras para } \\
\text { mantener un nivel adecuado en salud sexual } \\
\text { y reproductiva }\end{array}$ & 29 & 72,5 & 33 & 82,5 & $0,092^{a}$ \\
\hline $\begin{array}{l}\text { Manejar adecuadamente los problemas y } \\
\text { conflictos que pueden derivarse de la } \\
\text { vivencia de sexualidad }\end{array}$ & 32 & 80,0 & 29 & 72,5 & $0,403^{b}$ \\
\hline $\begin{array}{l}\text { Cambiar los comportamientos sexuales que } \\
\text { le permitan un crecimiento personal }\end{array}$ & 31 & 77,5 & 29 & 72,5 & $0,424^{a}$ \\
\hline $\begin{array}{l}\text { Abordar la realidad de la salud sexual y } \\
\text { reproductiva de forma novedosa y con } \\
\text { originalidad }\end{array}$ & 32 & 80,0 & 30 & 75,0 & $0,102^{b}$ \\
\hline $\begin{array}{l}\text { Cambiar comportamientos si es necesario, } \\
\text { para conservar la salud sexual y reproductiva. }\end{array}$ & 27 & 67,5 & 31 & 77,5 & $0,057^{a}$ \\
\hline
\end{tabular}

${ }^{a}$ Para prueba de Mc Nemar; ${ }^{b}$ Para prueba de Stuart Maswell; ${ }^{c}$ Para Mc Nemar a posteriori.

adolescente en todo el mundo favoreciendo los retos de las naciones con la salud de su población adolescente y adulta joven [11]

No obstante, cada país ha desarrollado sus apuestas interculturalizando el enfoque de manera que responda a las necesidades y características de sus adolescentes y jóvenes, pero siempre en miras a potenciar competencias psicosociales, como una estrategia para prevenir la enfermedad, posteriormente, para promover la salud, la formación ética, la educación sexual principalmente, entendida ella en su sentido amplio de desarrollo vital y no sólo biológico del ser humano [12].

La OMS, reconoce que son muchas las competencias necesarias para la vida, pero selecciona algunas como esenciales para hacer frente a los retos que presenta el 
Table 3

Estudiantes del grupo estudio que respondieron mucho al nivel conseguido en el desarrollo de habilidades para la vida sexual y reproductiva después de la intervención según variables exploradas.

\begin{tabular}{|c|c|c|}
\hline \multirow{2}{*}{$\begin{array}{l}\text { Nivel conseguido en el desarrollo de habilidades para la vida sexual } \\
\text { y reproductiva desarrolladasRespuesta: Mucho }\end{array}$} & \multicolumn{2}{|c|}{ Grupo estudio después } \\
\hline & No. & $\% n=40$ \\
\hline Incrementar sus conocimientos sobre temas de sexualidad & 39 & 97,5 \\
\hline $\begin{array}{l}\text { Cambiar para bien su forma de pensar sobre temas relacionados con } \\
\text { sexualidad }\end{array}$ & 39 & 97,5 \\
\hline $\begin{array}{l}\text { Tener más recursos para con seguridad cumplir su ideal en la salud } \\
\text { sexual y reproductiva }\end{array}$ & 39 & 97,5 \\
\hline $\begin{array}{l}\text { Ser más tolerante en su interacción con los demás y comprender } \\
\text { mejor sus vivencias sexuales }\end{array}$ & 37 & 92,5 \\
\hline $\begin{array}{l}\text { Expresar con claridad lo que piensa, lo que siente o necesita en } \\
\text { cuanto a su salud sexual y reproductiva }\end{array}$ & 40 & 100,0 \\
\hline $\begin{array}{l}\text { Ser capaz de manejar la presión de grupos de pares y mantener su } \\
\text { autonomía en las decisiones que se relacionan con su salud sexual y } \\
\text { reproductiva }\end{array}$ & 37 & 92,5 \\
\hline $\begin{array}{l}\text { Escoger alternativas más seguras para mantener un nivel adecuado en } \\
\text { su salud sexual y reproductiva }\end{array}$ & 40 & 100,0 \\
\hline $\begin{array}{l}\text { Manejar adecuadamente los problemas y conflictos que puedan } \\
\text { derivarse de la vivencia de la sexualidad. }\end{array}$ & 39 & 97,5 \\
\hline $\begin{array}{l}\text { Abordar la realidad de la salud sexual y reproductiva de forma } \\
\text { novedosa y con originalidad }\end{array}$ & 39 & 97,5 \\
\hline $\begin{array}{l}\text { Es una oportunidad de cambio en los comportamientos sexuales y de } \\
\text { crecimiento personal y social }\end{array}$ & 39 & 97,5 \\
\hline $\begin{array}{l}\text { Poner en práctica lo que ha aprendido y cambiar si es necesario sus } \\
\text { comportamientos para conservar su salud sexual y reproductiva }\end{array}$ & 39 & 97,5 \\
\hline $\begin{array}{l}\text { Recomendaría a otros estudiantes politécnicos participar en la } \\
\text { estrategia 'Sexualidad segura en tus manos' }\end{array}$ & 39 & 97,5 \\
\hline
\end{tabular}

mundo contemporáneo al cuidado de la vida, especialmente en el caso que nos ocupa para mantener y mejorar un nivel adecuado en la SSR de universitarios, estas habilidades son: Autoconocimiento, para incrementar los conocimientos sobre temas de sexualidad. Tener más recursos para con seguridad cumplir el ideal en la SSR, así como cambiar para bien la forma de pensar sobre temas relacionados con sexualidad. La empatía, para ser más tolerante en la interacción con los demás y comprender mejor las vivencias sexuales, la comunicación asertiva, para expresar con claridad lo que piensa, lo que siente o necesita en cuanto a la SSR, las relaciones interpersonales, para ser capaz de manejar la presión de grupos de pares y mantener autonomía en las decisiones que se relacionan con la SSR, la toma de decisiones, para escoger alternativas más seguras para mantener un nivel adecuado en la SSR, el manejo de problemas y conflictos, a fin de manejar adecuadamente los problemas y conflictos que pueden derivarse de la vivencia de la sexualidad, el pensamiento creativo, para abordar la realidad de la salud sexual y reproductiva de forma novedosa y con originalidad, y el 
pensamiento crítico para cambiar si es necesario sus comportamientos para conservar la SSR, que le permitan un crecimiento personal [13].

\section{Conclusiones}

Los resultados de la evaluación, obtenidos a través de las comparaciones de los grupos de estudio y control antes, grupo de control antes después y la medición posterior a la implementación de la estrategia educativa de las necesidades satisfechas de habilidades para la vida en el grupo de estudio, estuvieron dentro de lo esperado, ya que se observó que la mayoría de variables estudiadas respecto a la necesidad de desarrollar habilidades para la vida son homogéneas en el grupo de estudio y control antes de implementar la estrategia; además de que no se encontraron diferencias significativas en la comparación del grupo control antes y después.

La medición posterior a la implementación de la estrategia respecto a necesidades satisfechas o nivel conseguido en el desarrollo de habilidades para la vida sexual y reproductiva en el grupo de estudio fue elevado, incluso para algunas de ellas, el 100\% de estudiantes apreció que lo había logrado.

\section{Agradecimientos}

Expresamos un agradecimiento a los estudiantes de la Escuela Superior Politécnica de Chimborazo por su colaboración en las distintas etapas de investigación.

\section{Conflicto de Interés}

No existen intereses particulares por parte de los autores, de la entidad científica o financiadora que pudiesen afectar directa o indirectamente a los resultados obtenidos.

\section{Limitación de Responsabilidad}

Declaro que todos los puntos de vista expresados son de entera responsabilidad de los autores.

\section{Fuentes de Apoyo}

Fondos propios y ayuda económica de la ESPOCH, para estudios de Doctorado.

\section{References}

[1] Cunias M, Ramos M, López Y. Nivel de educación sexual universitaria:¿ utopía o realidad?. HacedorAIAPAEC. 2019;3(2):[aprox. 8 p.].

[2] Gómez D, Ochoa M, et al. Salud sexual y reproductiva en estudiantes universitarios de una institución de educación superior en Colombia. Revista de Salud Pública. 2014;16: 
[3] Ordoñez J, Real J, Gallardo J, Alvarado H, Roby A. Conocimientos sobre salud sexual y su relación con el comportamiento sexual en estudiantes universitarios. Anales de la Facultad de medicina. 2017;78(4)

[4] Hernández Y, et al. Creencias, actitudes y prácticas en sexualidad en estudiantes de psicología clínica de la Universidad Católica de Cuenca. Revista Electrónica de Psicología Iztacala. 2018;20(3):

[5] Constitución de la República del Ecuador 2008 No. 449 (Ecuador).

[6] Orientaciones Técnicas Internacionales sobre Educación en Sexualidad. Un enfoque basado en evidencia orientado a escuelas, docentes y educadores de la salud. Vol. 1. Santiago de Chile: UNESCO; 2010. Justificación de la educación en sexualidad, p. 1-27.

[7] Organización Mundial de la Salud. Enfoque de habilidades para la vida para un desarrollo saludable de niños y adolescentes. Ginebra: OMS; 2001.

[8] Cantor M, Pérez E, Carrillo S. Revista AiBi. Redes sociales e identidad social. 2018;6(1):

[9] Rizo M, Velandia M, Noreña A, Cortés E. La epidemiología como fundamento del diseño curricular de enfermería en salud sexual y reproductiva de los adolescentes y jóvenes. Ciencia e innovación en Salud. 2018;

[10] Organización Mundial de la Salud. Serie de información de la OMS sobre salud escolar. Salud OM editor. Ginebra: OMS; 2003. Creación de un entorno para el bienestar emocional y social: Una responsabilidad importante de una escuela que promueva la salud y sea amigable para los niños.

[11] Carrillo S, et al. Habilidades para la vida como elementos en la promoción y educación para la salud en los adolescentes y jóvenes. Revista vft. 2018;37(2)

[12] Martínez V. Habilidades para la Vida: Una propuesta de formación humana. Itinerario Educativo. 2014;28(63)

[13] Saeteros R, Pérez J, Sanabria G. Estrategia de educación sexual con metodología de pares para estudiantes universitarios ecuatorianos. Revista Cubana Salud Pública. 2018;44(2): 